\title{
Multishelled NiO Hollow Spheres Decorated by Graphene Nanosheets as Anodes for Lithium-Ion Batteries with Improved Reversible Capacity and Cycling Stability
}

\author{
Lihua Chu, ${ }^{1}$ Meicheng Li, ${ }^{1,2}$ Yu Wang, ${ }^{1}$ Xiaodan Li, ${ }^{1}$ Zipei Wan, \\ Shangyi Dou, ${ }^{1}$ and Yue Chu ${ }^{1}$ \\ ${ }^{1}$ State Key Laboratory of Alternate Electrical Power System with Renewable Energy Sources, \\ North China Electric Power University, Beijing 102206, China \\ ${ }^{2}$ Chongqing Materials Research Institute, Chongqing 400707, China \\ Correspondence should be addressed to Meicheng Li; mcli@ncepu.edu.cn
}

Received 15 December 2015; Revised 16 January 2016; Accepted 21 April 2016

Academic Editor: Raul Arenal

Copyright ( $) 2016$ Lihua Chu et al. This is an open access article distributed under the Creative Commons Attribution License, which permits unrestricted use, distribution, and reproduction in any medium, provided the original work is properly cited.

Graphene-based nanocomposites attract many attentions because of holding promise for many applications. In this work, multishelled $\mathrm{NiO}$ hollow spheres decorated by graphene nanosheets nanocomposite are successfully fabricated. The multishelled $\mathrm{NiO}$ microspheres are uniformly distributed on the surface of graphene, which is helpful for preventing aggregation of asreduced graphene sheets. Furthermore, the $\mathrm{NiO}$ /graphene nanocomposite shows much higher electrochemical performance with a reversible capacity of $261.5 \mathrm{mAh} \mathrm{g}^{-1}$ at a current density of $200 \mathrm{~mA} \mathrm{~g}^{-1}$ after 100 cycles tripled compared with that of pristine multishelled $\mathrm{NiO}$ hollow spheres, implying the potential application in modern science and technology.

\section{Introduction}

With the rapid development of the global economy, fast depletion of nonrenewable energy, and the increasing environmental problems, it is urgent to develop new clean and sustainable sources and technologies for energy storage and conversion with high efficiency [1-3]. Rechargeable lithiumion batteries (LIBs) are a promising one for the upcoming demand such as electric vehicles and grid storage systems because of their high energy density, long lifespan, and environmental benignancy.

As one of the special structures of carbon, graphene has initiated enormous scientific activities, with the honeycomb lattice structure, unique structural features of chemical stability, high surface area, flexibility, and superior electric and thermal conductivity. It has been used as ideal building blocks for energy storage systems with improved electrochemical properties compared with these host materials, such as metal, metal oxide, and sulfide $[4,5]$. To further explore the potential application of graphene-based nanomaterials, deriving from the decoration of graphene nanosheets (GNS) with nanomaterials attracts more and more interest. So far, several graphene-based nanocomposites have been successfully fabricated and show desirable combinations of properties that are not found in individual components $[6,7]$.

Nickel oxide $(\mathrm{NiO})$ is one of prospective anode materials in li-ion battery (LIB), which possesses the advantages of high theoretical capacity $\left(717 \mathrm{mAh} \mathrm{g}^{-1}\right)$, high natural abundance, nontoxicity, low cost, and environmental benignity [8-11]. However, the practical utilization of $\mathrm{NiO}$ is still limited owing to its poor cycling and rate performances of the NiO-based LIBs. Several strategies have been undertaken to enhance the performance of $\mathrm{NiO}$ electrode materials. One wellestablished method is constructing hybrid with GNS. Various structures of $\mathrm{NiO} / \mathrm{GNS}$, such as porous $\mathrm{NiO}$-wrapped GNS [12], 3D-hierarchical NiO/GNS [13], and graphene anchored with nickel nanoparticles [14], have been prepared, revealing that the NiO/GNS nanocomposite reinforces the properties of pristine $\mathrm{NiO}$ which favors their wide application in LIBs. 


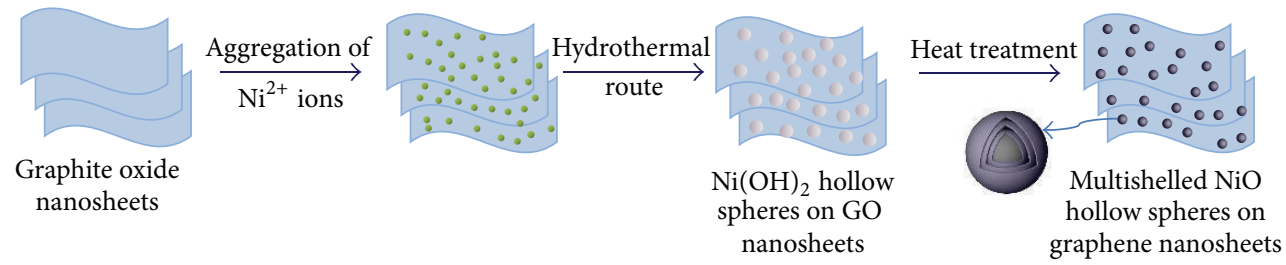

FIGURE 1: Illustration of the procedures for preparation of $\mathrm{NiO} /$ graphene hierarchical structure.

In this work, we report the solvothermal method for the synthesis of multishelled NiO hollow spheres/GNS nanocomposite, in which the multishelled $\mathrm{NiO}$ hollow spheres are wrapped by GNS. This hybrid architecture is found to display much improved electrochemical properties by comparison to that of pristine multishelled $\mathrm{NiO}$ hollow microspheres.

\section{Experimental}

The composite was prepared by the solvothermal method. $3 \mathrm{~mL}$ graphite oxide $\left(6 \mathrm{~mol} \mathrm{~L}^{-1}\right)$ was ultrasonically dispersed in $30 \mathrm{~mL}$ of deionized water and then $1 \mathrm{~g}$ of $\mathrm{Ni}(\mathrm{NO})_{3} \cdot 6 \mathrm{H}_{2} \mathrm{O}$ and $1 \mathrm{~g}$ of $\mathrm{D}$-glucose were added, adjusting the $\mathrm{pH}$ to 10.90 with $\mathrm{NH}_{3} \cdot \mathrm{H}_{2} \mathrm{O}$, using a $\mathrm{pH}$ meter and magnetic stirrer to form a homogeneous solution at room temperature. The solution was then transferred into a Teflon-lined stainless steel autoclave $(50 \mathrm{~mL})$, sealed, and heated at $150^{\circ} \mathrm{C}$ for $15 \mathrm{~h}$. The product was collected by centrifugation, washed with deionized water several times to remove impurity, and then dried at $60^{\circ} \mathrm{C}$ in vacuum. Finally, the $\mathrm{NiO} /$ graphene was obtained by calcining the precursor in $\mathrm{N}_{2}$ at $300^{\circ} \mathrm{C}$ for $2 \mathrm{~h}$ and then in air at $500^{\circ} \mathrm{C}$ for $2 \mathrm{~h}$. For comparison, the multishelled $\mathrm{NiO}$ spheres were prepared by a similar solution-based method and subsequent calcination with the absence of graphene oxide (GO).

The crystallographic structures, morphology, and microstructure of the as-prepared products were characterized by Bruker D8 Focus X-ray powder diffractometer with using $\mathrm{Cu} \mathrm{K}_{\alpha}(\lambda=1.5406 \AA)$ radiation over a range of $2 \theta$ from $20^{\circ}$ to $90^{\circ}$, FEI Quanta $200 \mathrm{~F}$ microscope field emission scanning electron microscope (FESEM), and Tecnai G2 F20 Field emission transmission electron microscopy (TEM) with accelerating voltage of $200 \mathrm{kV}$. Raman measurements were recorded on Laser Confocal Micro-Raman Spectroscopy (LabRAM Aramis).

Electrochemical measurements were conducted using a CR-2032-coin type cell configuration. The electrode materials are a slurry consisting of $80 \mathrm{wt} \%$ of electrode materials, $10 \mathrm{wt} \%$ of acetylene black (Super-P), and $10 \mathrm{wt} \%$ of polyvinylidene fluoride (PVDF) dissolved in N-methyl-2pyrrolidinone. They were coated onto a copper foil and dried at $100^{\circ} \mathrm{C}$ in vacuum for $12 \mathrm{~h}$ before pressing. Lithium foil was used as the counter and reference electrodes and the polypropylene foil (Celgard 2400) was used as the separator. The electrolyte was $1 \mathrm{M} \mathrm{LiPF}_{6}$ dissolved in a mixture of ethylene carbonate (EC) and dimethyl carbonate (DMC) (v/v $=1: 1)$. Galvanostatic discharge-charge (GDC) experiments were carried out at different current densities in the voltage range of $0.01-3.00 \mathrm{~V}$ with a multichannel battery tester (Maccor, Inc., USA). Cycling and rate performances were measured by the electrochemical workstation (LAND battery test system).

\section{Results and Discussion}

A schematic illustration of the fabrication process of $\mathrm{NiO} /$ graphene composite is shown in Figure 1. Graphite oxide (GO) was sonicated in di-water to form a homogeneous suspension. Then, the $\mathrm{GO}$ nanosheets absorb $\mathrm{Ni}^{2+}$ ions, which react with $\mathrm{OH}^{-}$to grow $\mathrm{Ni}(\mathrm{OH})_{2}$ architectures on GO nanosheets. Here, $\mathrm{OH}^{-}$were released by $\mathrm{NH}_{3} \cdot \mathrm{H}_{2} \mathrm{O}$, which can also control the morphology of the $\mathrm{Ni}(\mathrm{OH})_{2}$ architectures. The formation of $\mathrm{Ni}(\mathrm{OH})_{2}$ from $\mathrm{Ni}^{2+}$ ions and $\mathrm{NH}_{3} \cdot \mathrm{H}_{2} \mathrm{O}$ can be expressed by the following reactions:

$$
\begin{aligned}
& \mathrm{Ni}^{2+}+x \mathrm{NH}_{3} \cdot \mathrm{H}_{2} \mathrm{O} \longrightarrow\left[\mathrm{Ni}\left(\mathrm{NH}_{3}\right)_{x}\right]^{2+}+6 \mathrm{H}_{2} \mathrm{O} \\
& {\left[\mathrm{Ni}\left(\mathrm{NH}_{3}\right)_{x}\right]^{2+} \longrightarrow \mathrm{Ni}^{2+}+x\left(\mathrm{NH}_{3}\right) } \\
& \mathrm{NH}_{3} \cdot \mathrm{H}_{2} \mathrm{O} \longrightarrow \mathrm{NH}_{4}^{+}+\mathrm{OH}^{-} \\
& \mathrm{Ni}^{2+}+2 \mathrm{OH}^{-} \longrightarrow \mathrm{Ni}(\mathrm{OH})_{2}
\end{aligned}
$$

During the calcination, $\mathrm{Ni}(\mathrm{OH})_{2}$ decomposed to yield multishelled $\mathrm{NiO}$ hollow spheres following $\mathrm{Ni}(\mathrm{OH})_{2} \rightarrow$ $\mathrm{NiO}+\mathrm{H}_{2} \mathrm{O}$, and $\mathrm{GO}$ nanosheets reduced to graphene by losing oxygen-containing surface groups [15, 16]. So the $\mathrm{Ni}(\mathrm{OH})_{2} / \mathrm{GO}$ precursor converted to $\mathrm{NiO} / \mathrm{GNS}$ composites after annealing. The pristine multishelled $\mathrm{NiO}$ hollow spheres would be obtained without GO during the similar reaction process, which were discussed in our previous paper [17].

The XRD patterns of $\mathrm{NiO}$ and $\mathrm{NiO} / \mathrm{GNS}$ composite are shown in Figure 2(a). All of the diffraction peaks of pure $\mathrm{NiO}$ sample can be ascribed to face centered cubic NiO (JCPDS number 071-1179), which correspond to (111), (200), (220), (311), and (222) planes, respectively. All the characteristic peaks of pure $\mathrm{NiO}$ are also observed for $\mathrm{NiO} / \mathrm{GNS}$ composite. (002) diffraction peak of GNS is typically located at about $24^{\circ}$ in the XRD pattern, but it is not obvious in Figure 2(a). Because the content of graphene is low and the diffraction of disorderedly stacked GNS is quite weaker as compared to the well-crystalline $\mathrm{NiO}$, the presence of graphene can be confirmed in the Raman spectra of NiO/GNS, as shown in Figure 2(b), where the $G$ characteristic peaks of graphene are obvious, though the $\mathrm{D}$ peak located at $\sim 1350 \mathrm{~cm}^{-1}$ is invisible.

The morphology of pristine $\mathrm{NiO}$ and $\mathrm{NiO} / \mathrm{GNS}$ composite is compared by SEM and TEM. Pure NiO is composed of 


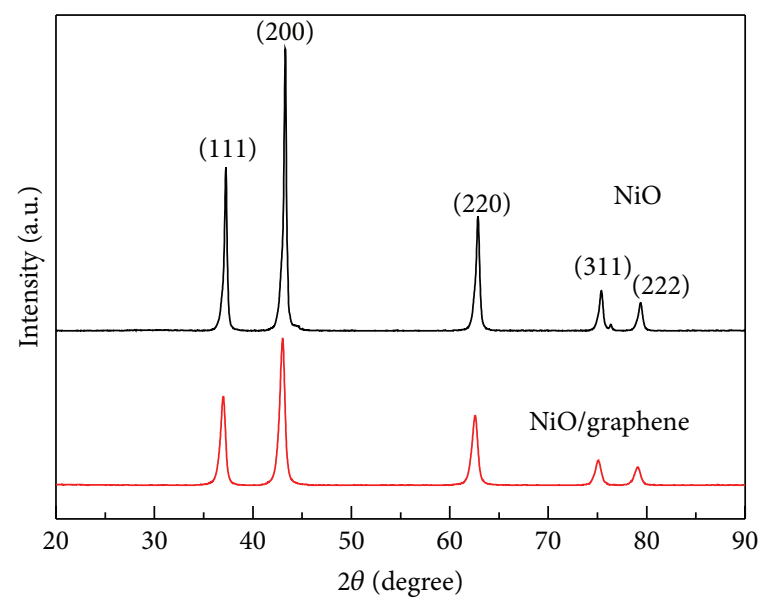

(a)

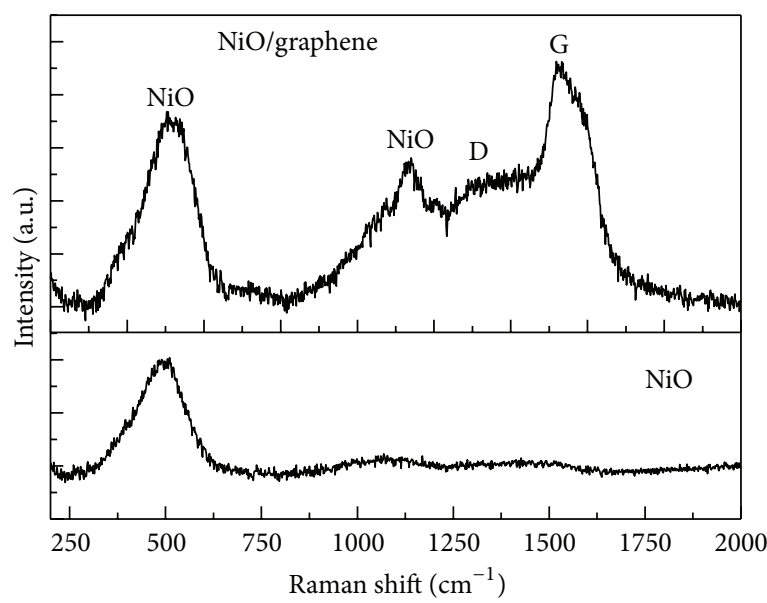

(b)

Figure 2: (a) X-ray diffraction patterns and (b) Raman spectra for pristine $\mathrm{NiO}$ and $\mathrm{NiO}$ /graphene composite.

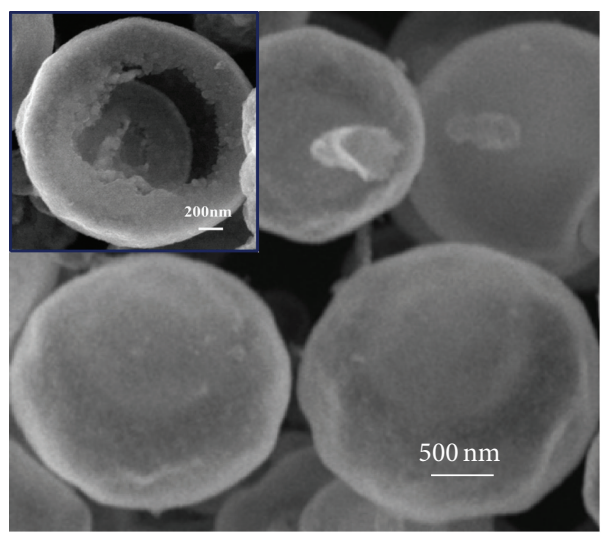

(a)

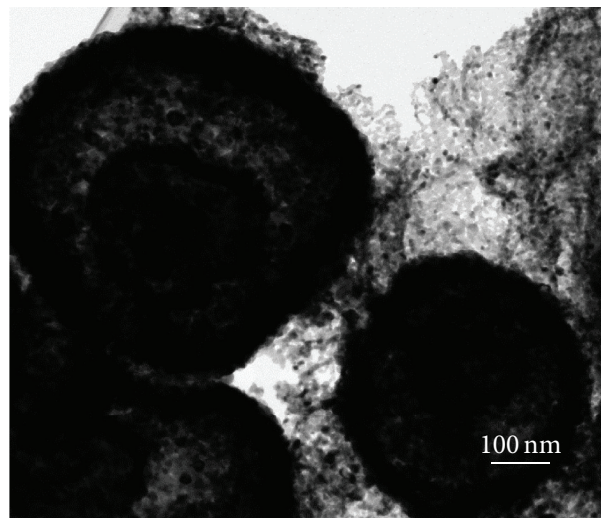

(c)

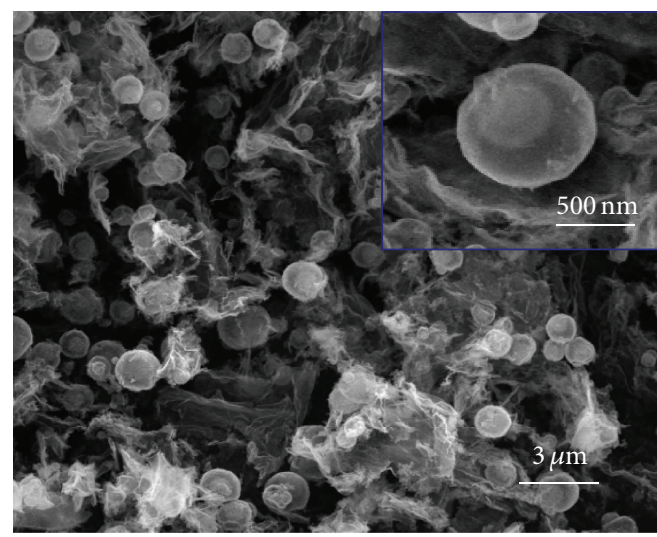

(b)

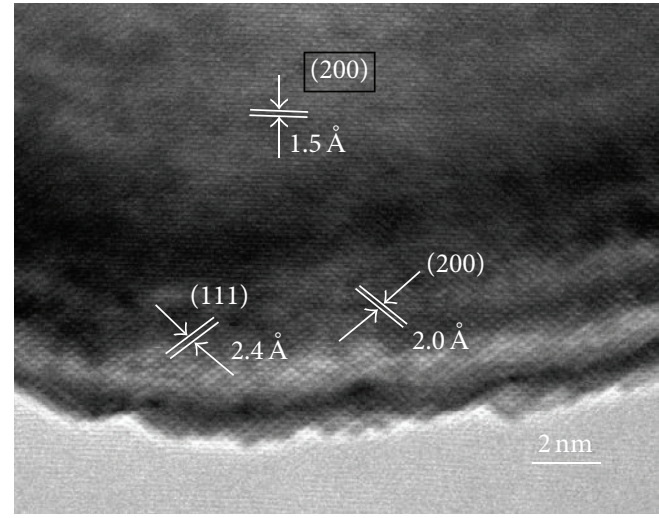

(d)

FIGURE 3: SEM images of pristine multishelled NiO spheres (a), NiO/graphene (b), TEM images of NiO/graphene (c), and HRTEM pattern of multishelled $\mathrm{NiO}$ spheres (d).

multishelled spheres as shown in Figure 3(a). The NiO/GNS are composed of typical rippled and crumpled GNS and multishelled $\mathrm{NiO}$ spheres (Figure 3(b)). It can be clearly seen that the multishelled $\mathrm{NiO}$ microspheres with diameter varying from $2.0 \mu \mathrm{m}$ to $3.5 \mu \mathrm{m}$ are uniformly distributed on the surface of GNS. The hybrid architecture can effectively prevent the agglomeration of as-reduced GNS. The inset of Figure $3(\mathrm{~b})$ is the multishelled $\mathrm{NiO}$ sphere on the GNS which demonstrates that the multishelled $\mathrm{NiO}$ spheres do not change after the addition of graphene. Figure 3(c) shows 


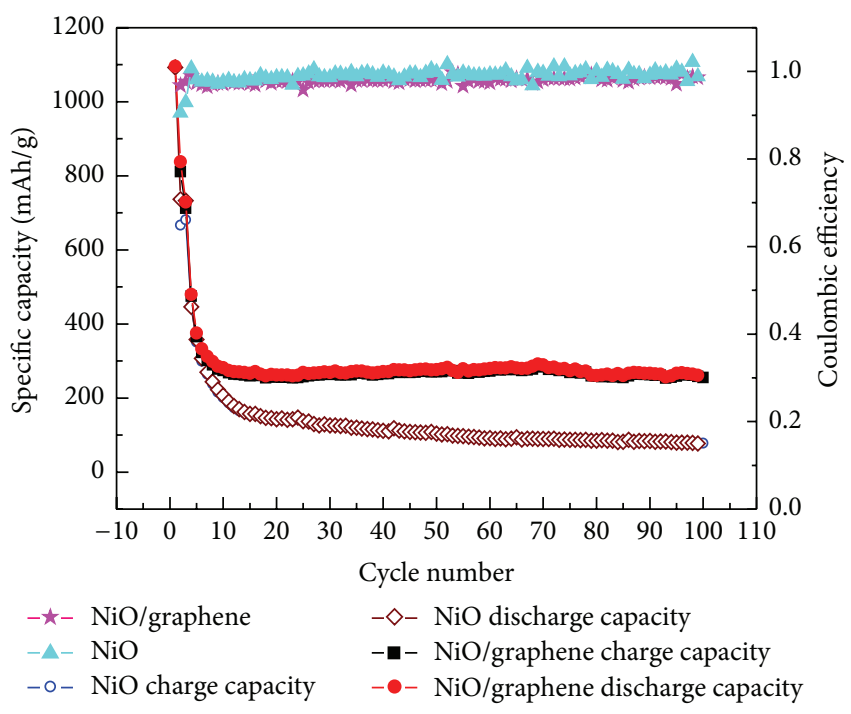

(a)

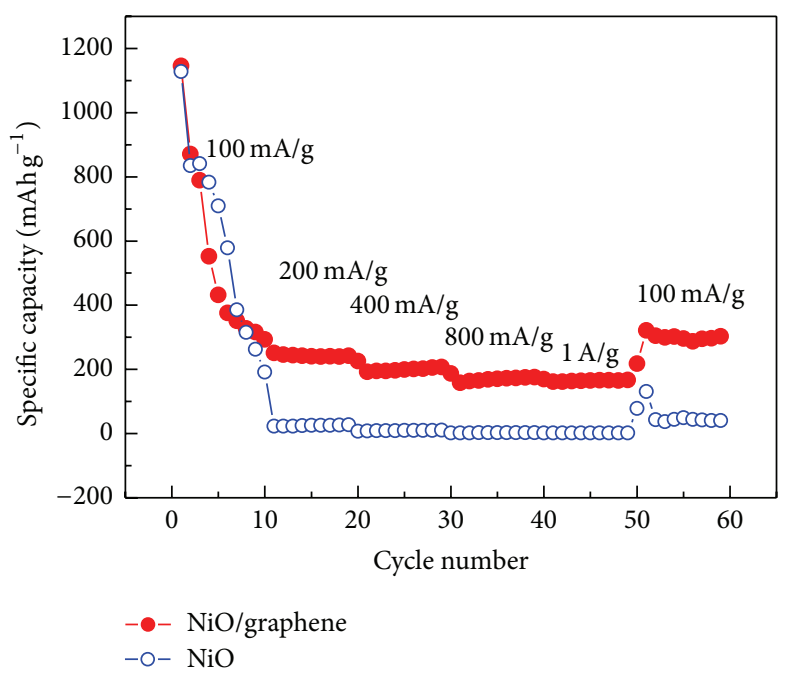

(b)

FIgURE 4: (a) Capacity retention and Coulombic efficiency at a current density of $200 \mathrm{mAg}^{-1}$. (b) Rate performance at various current densities between $100 \mathrm{mAg}^{-1}$ and $1 \mathrm{Ag}^{-1}$.

the TEM image of $\mathrm{NiO} / \mathrm{GNS}$ which further confirms the multishelled sphere feature of NiO. The HRTEM pattern of multishelled $\mathrm{NiO}$ sphere is presented in Figure 3(d). The lattice spacing of $0.15,0.2$, and $0.24 \mathrm{~nm}$ is observed, corresponding to the interspaces of (220), (200) and (111), and (200) planes of cubic NiO.

The electrochemical properties of $\mathrm{NiO} / \mathrm{GNS}$ composite were evaluated as anode for lithium-ion batteries (LIBs). Figure 4 displays the cycling and rate performances of $\mathrm{NiO} / \mathrm{GNS}$ composite and pristine $\mathrm{NiO}$. The initial discharge capacities are $\sim 1100 \mathrm{mAh} \mathrm{g}^{-1}$ as shown in Figure 4(a). It is much higher than the theoretical value $\left(718 \mathrm{mAhg}^{-1}\right)$, mainly attributed to the solid electrolyte interphase (SEI) film formation, and the additional $\mathrm{Li}^{+}$storage in the space between $\mathrm{NiO}$ spheres and GNS. The reversible capacity of $\mathrm{NiO}$ spheres decreases to $77.1 \mathrm{mAh} \mathrm{g}^{-1}$ after 100 cycles. While the reversible capacity of $\mathrm{NiO} / \mathrm{GNS}$ composite still can retain $261.5 \mathrm{mAh} \mathrm{g}^{-1}$ after 100 cycles, which is more than three times of pristine $\mathrm{NiO}$ sphere. The columbic efficiencies increase to and almost keep stable in the scale of $98-99 \%$ at successive cycles, indicating that the formed SEI film is favorable and stable [18]. The enhanced cycling performance of $\mathrm{NiO} / \mathrm{GNS}$ composite should be ascribed to the addition of graphene and the hybrid architecture. Firstly, the GNS have much better conductivity due to the wider layer spacing compared with $\mathrm{NiO}$. The $d$-spacing of GNS was found to be $0.365 \mathrm{~nm}$, and the maximum $d$-spacing of $\mathrm{NiO}$ is $0.24 \mathrm{~nm}$ ((111) lattice plane) [19]. Therefore, the GNS can offer additional sites for accommodation of $\mathrm{Li}^{+}$leading to the enhanced reversible capacity. Secondly, the flexible GNS can accommodate the volume change and prevent the aggregation of active materials upon cycling. Thirdly, the hybrid architecture can also be helpful for preventing aggregating or restacking of as-reduced GNS.
Moreover, the NiO/GNS composites also exhibit enhanced rate capacity compared with pristine $\mathrm{NiO}$ spheres, as presented in Figure 4(b). The discharge capacities of $\mathrm{NiO} / \mathrm{GNS}$ at $100 \mathrm{mAg}^{-1}, 200 \mathrm{~mA} \mathrm{~g}^{-1}, 400 \mathrm{mAg} \mathrm{g}^{-1}$, $800 \mathrm{mAg}^{-1}$, and $1 \mathrm{Ag}^{-1}$ are $292.3 \mathrm{mAhg}^{-1}$ (10th cycle), $225.9 \mathrm{mAhg}^{-1}$ (20th cycle), $187.3 \mathrm{mAhg}^{-1}$ (30th cycle), $169.3 \mathrm{mAh} \mathrm{g}^{-1}$ (40th cycle), and $166.5 \mathrm{mAh} \mathrm{g}^{-1}$ (50th cycle), respectively. In the initial 10 cycles with a small current density $\left(100 \mathrm{mAg}^{-1}\right)$, the capacities of pristine $\mathrm{NiO}$ and $\mathrm{NiO} / \mathrm{GNS}$ composite are comparable, and the pristine $\mathrm{NiO}$ is even a little better than $\mathrm{NiO} / \mathrm{GNS}$ composite, which may be caused by the lower capacity of graphene as compared to $\mathrm{NiO}$ [18]. However, the situation reverses when the current density increases. The enhanced rate capacity could be reasonably attributed to advantageous combination of the highly conductive GNS and the favorable multishelled $\mathrm{NiO}$ hollow spheres architecture with large specific surface area and open inner cavity, which can facilitate the rapid diffusion of lithium ions from electrolyte to active material.

As shown in Figure 4(b), the capacity dropped dramatically from $\sim 1100 \mathrm{mAh} \mathrm{g}^{-1}$ to a little more than $300 \mathrm{mAh} \mathrm{g}^{-1}$ during the first five cycles with a small current density $\left(100 \mathrm{~mA} \mathrm{~g}^{-1}\right)$. Generally, the capacity lost in the first several cycles can be attributed to the irreversible reactions involved in the formation of the SEI layer. In addition, the formation and stabilization of the SEI layer are a gradual process, so that stable capacity also requires a process, which has been described in many works [20,21]. It is also probably caused by large theoretical capacity difference between $\mathrm{NiO}$ $\left(717 \mathrm{mAh} \mathrm{g}^{-1}\right)$ and graphene $\left(372 \mathrm{mAh} \mathrm{g}^{-1}\right)$. During the first several cycles, the synergy effect between $\mathrm{NiO}$ and graphene does not play well during the lithiation and delithiation process; therefore, the $\mathrm{NiO}$ and graphene do not serve well in 
terms of improving and stabilizing capacity. So the dropped capacity is due to the formation of SEI layer and the capacity difference in the first several cycles.

The NiO/GNS composite demonstrates improved electrochemical performance, which could be due to several reasons. Firstly, the wider GNS can offer additional sites for the accommodation of $\mathrm{Li}^{+}$compared with $\mathrm{NiO}$. Moreover, the well-contact GNS and multishelled $\mathrm{NiO}$ hollow spheres can facilitate the continuous and rapid conducting pass way of $\mathrm{Li}^{+}$, which are beneficial for enhancing specific capacity and rate performance of the active materials. Secondly, GNS are anticipated to prevent the collapse and aggregation of the active materials upon cycling, which brings about excellent cycling stability. Vice versa, the hybrid architecture can also be helpful for preventing aggregating of GNS [12]. Thirdly, the large specific surface area and open inner cavity of GNS and the favorable multishelled $\mathrm{NiO}$ hollow spheres architecture can provide sufficient electrode/electrolyte contact areas and facilitate the continuous and rapid electron transport through the electrodes, resulting in the improved rate performance.

\section{Conclusions}

In summary, we have successfully fabricated $\mathrm{NiO} / \mathrm{GNS}$ composite, in which the multishelled $\mathrm{NiO}$ microspheres are uniformly distributed on the surface of GNS. The composite shows much enhanced cycling stability and capacity compared with the pristine multishelled $\mathrm{NiO}$ hollow spheres, when evaluated as the anode materials in LIBs. The superior performance in LIBs originates from the addition of GNS and the sandwich-like architecture, which can facilitate the continuous and rapid electron transport and prevent the collapse of nanostructures and aggregation of the active materials. The strategies described here could offer an effective method to improve the electrochemical performance of other electrode materials with large volume changes and low electrical conductivities.

\section{Competing Interests}

The authors declare that they have no competing interests.

\section{Acknowledgments}

This work is supported partially by National High-tech R\&D Program of China (863 Program, no. 2015AA034601), National Natural Science Foundation of China (Grant nos. 91333122, 51402106, 51372082, 51172069, 61204064, and 51202067), Ph.D. Programs Foundation of Ministry of Education of China (Grant nos. 20120036120006 and 20130036110012), Par-Eu Scholars Program, and the Fundamental Research Funds for the Central Universities.

\section{References}

[1] H. Xia, C. Hong, X. Shi et al., "Hierarchical heterostructures of $\mathrm{Ag}$ nanoparticles decorated $\mathrm{MnO}_{2}$ nanowires as promising electrodes for supercapacitors," Journal of Materials Chemistry A, vol. 3, no. 3, pp. 1216-1221, 2015.
[2] H. Xia, C. Hong, B. Li et al., "Facile synthesis of hematite quantum dot/functionalized graphene-sheet composites as advanced anode materials for asymmetric supercapacitors," Advanced Functional Materials, vol. 25, no. 4, pp. 627-635, 2015.

[3] H. Xia, D. Zhu, Z. Luo et al., "Hierarchically structured $\mathrm{Co}_{3} \mathrm{O}_{4} @ \mathrm{Pt} @ \mathrm{MnO}_{2}$ nanowire arrays for high-performance supercapacitors," Scientific Reports, vol. 3, article 2978, 2013.

[4] J. Zhu, D. Yang, Z. Yin, Q. Yan, and H. Zhang, "Graphene and graphene-based materials for energy storage applications," Small, vol. 10, no. 17, pp. 3480-3498, 2014.

[5] M. Pumera, "Graphene-based nanomaterials for energy storage," Energy and Environmental Science, vol. 4, no. 3, pp. 668$674,2011$.

[6] C. Zhang, X. Peng, Z. Guo et al., "Carbon-coated $\mathrm{SnO}_{2}$ /graphene nanosheets as highly reversible anode materials for lithium ion batteries," Carbon, vol. 50, no. 5, pp. 1897-1903, 2012.

[7] D.-H. Lee, J.-C. Kim, H.-W. Shim, and D.-W. Kim, "Highly reversible li storage in hybrid $\mathrm{NiO} / \mathrm{Ni} /$ graphene nanocomposites prepared by an electrical wire explosion process," ACS Applied Materials \& Interfaces, vol. 6, no. 1, pp. 137-142, 2014.

[8] N. S. Spinner, A. Palmieri, N. Beauregard, L. Zhang, J. Campanella, and W. E. Mustain, "Influence of conductivity on the capacity retention of $\mathrm{NiO}$ anodes in Li-ion batteries," Journal of Power Sources, vol. 276, pp. 46-53, 2015.

[9] X. Yan, X. Tong, J. Wang, C. Gong, M. Zhang, and L. Liang, "Synthesis of hollow nickel oxide nanotubes by electrospinning with structurally enhanced lithium storage properties," Materials Letters, vol. 136, pp. 74-77, 2014.

[10] W. Sun, X. Rui, J. Zhu et al., "Ultrathin nickel oxide nanosheets for enhanced sodium and lithium storage," Journal of Power Sources, vol. 274, pp. 755-761, 2015.

[11] D. Mao, J. Yao, X. Lai, M. Yang, J. Du, and D. Wang, "Hierarchically mesoporous hematite microspheres and their enhanced formaldehyde-sensing properties," Small, vol. 7, no. 5, pp. 578$582,2011$.

[12] D. Xie, Q. Su, W. Yuan, Z. Dong, J. Zhang, and G. Du, "Synthesis of porous $\mathrm{NiO}$-wrapped graphene nanosheets and their improved lithium storage properties," Journal of Physical Chemistry C, vol. 117, no. 46, pp. 24121-24128, 2013.

[13] L. Tao, J. Zai, K. Wang et al., "3D-hierarchical NiO-graphene nanosheet composites as anodes for lithium ion batteries with improved reversible capacity and cycle stability," RSC Advances, vol. 2, no. 8, pp. 3410-3415, 2012.

[14] Y. J. Mai, J. P. Tu, C. D. Gu, and X. L. Wang, "Graphene anchored with nickel nanoparticles as a high-performance anode material for lithium ion batteries," Journal of Power Sources, vol. 209, pp. $1-6,2012$.

[15] H. Li, L. Pan, C. Nie, Y. Liu, and Z. Sun, "Reduced graphene oxide and activated carbon composites for capacitive deionization," Journal of Materials Chemistry, vol. 22, no. 31, pp. 1555615561, 2012.

[16] I. R. M. Kottegoda, N. H. Idris, L. Lu, J.-Z. Wang, and H.K. Liu, "Synthesis and characterization of graphene-nickel oxide nanostructures for fast charge-discharge application," Electrochimica Acta, vol. 56, no. 16, pp. 5815-5822, 2011.

[17] L. Chu, M. Li, Z. Wan et al., "Morphology control and fabrication of multi-shelled $\mathrm{NiO}$ spheres by tuning the $\mathrm{pH}$ value via a hydrothermal process," CrystEngComm, vol. 16, no. 48, pp. 11096-11101, 2014.

[18] Y. Huang, X.-L. Huang, J.-S. Lian, D. Xu, L.-M. Wang, and X.-B. Zhang, "Self-assembly of ultrathin porous $\mathrm{NiO}$ 
nanosheets/graphene hierarchical structure for high-capacity and high-rate lithium storage," Journal of Materials Chemistry, vol. 22, no. 7, pp. 2844-2847, 2012.

[19] E. J. Yoo, J. Kim, E. Hosono, H.-S. Zhou, T. Kudo, and I. Honma, "Large reversible Li storage of graphene nanosheet families for use in rechargeable lithium ion batteries," Nano Letters, vol. 8, no. 8, pp. 2277-2282, 2008.

[20] X. Huang, H. Yu, J. Chen, Z. Lu, R. Yazami, and H. H. Hng, "Ultrahigh rate capabilities of lithium-ion batteries from 3D ordered hierarchically porous electrodes with entrapped active nanoparticles configuration," Advanced Materials, vol. 26, no. 8, pp. 1296-1303, 2014.

[21] K. Cao, L. Jiao, H. Liu et al., "3D hierarchical porous $\alpha$ $\mathrm{Fe}_{2} \mathrm{O}_{3}$ nanosheets for high-performance lithium-ion batteries," Advanced Energy Materials, vol. 5, no. 4, Article ID 1401421, 2015. 

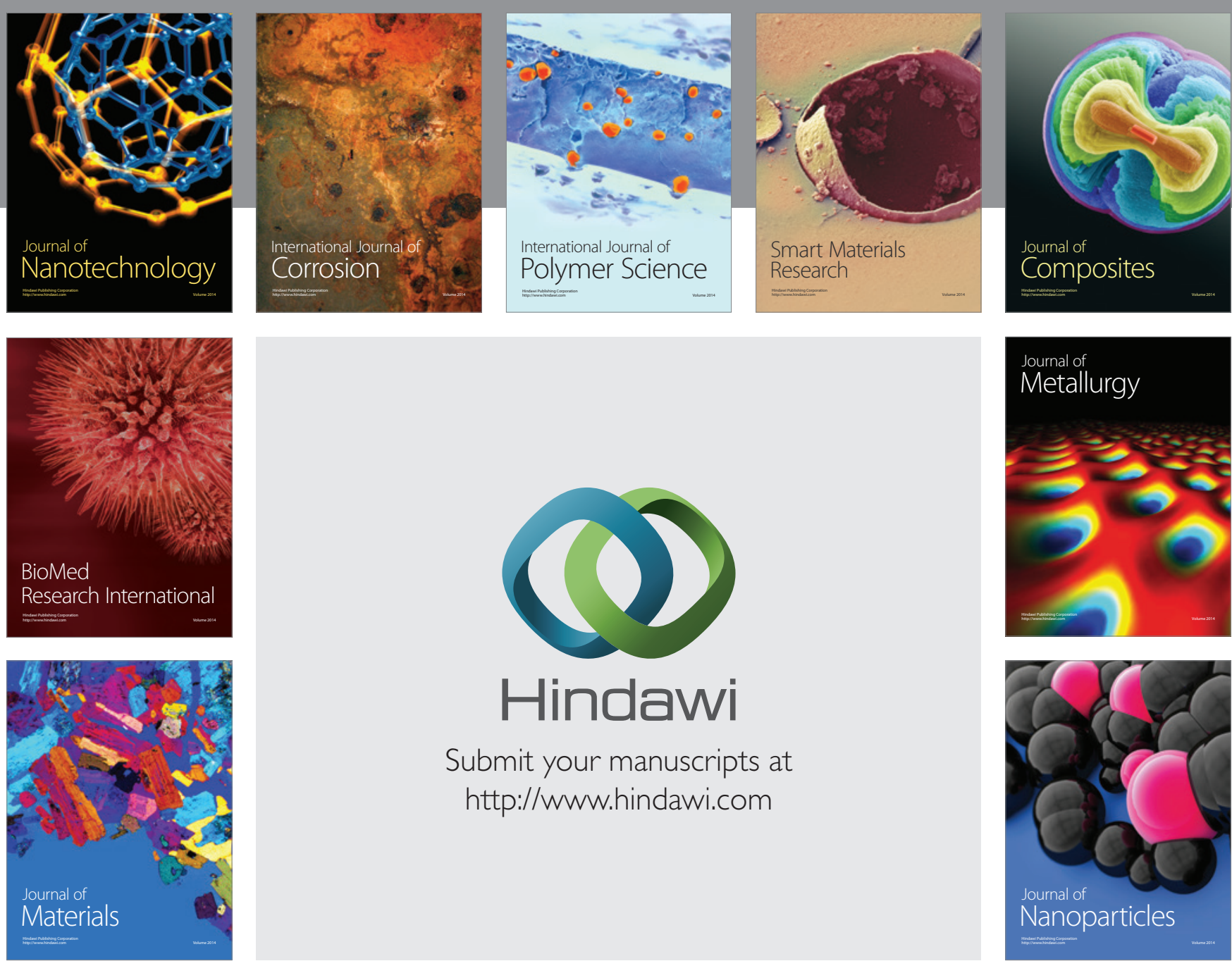

\section{Hindawi}

Submit your manuscripts at

http://www.hindawi.com

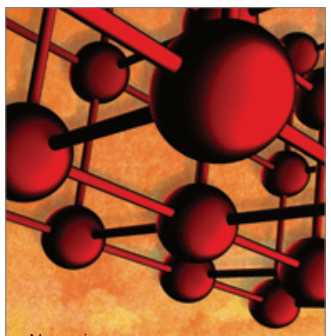

Materials Science and Engineering
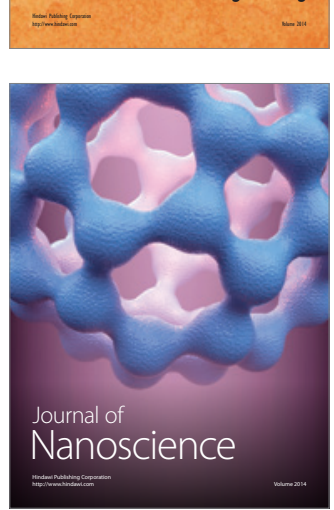
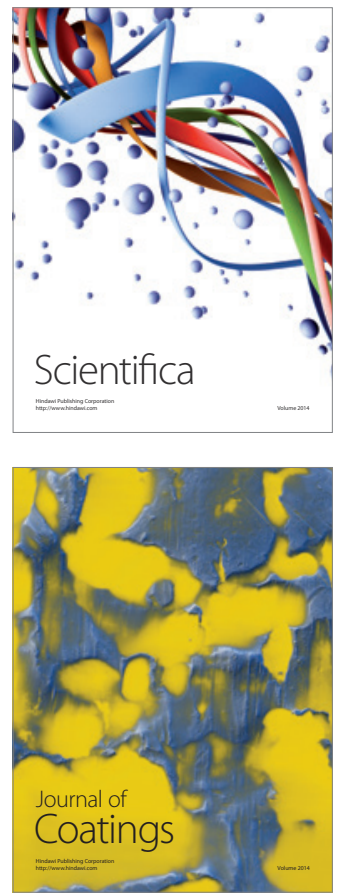
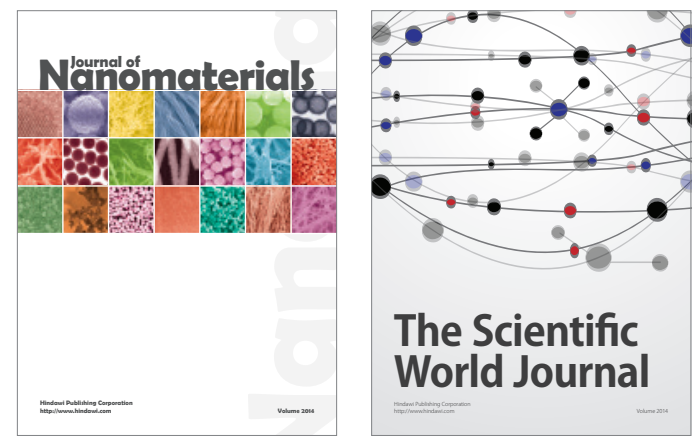

The Scientific World Journal
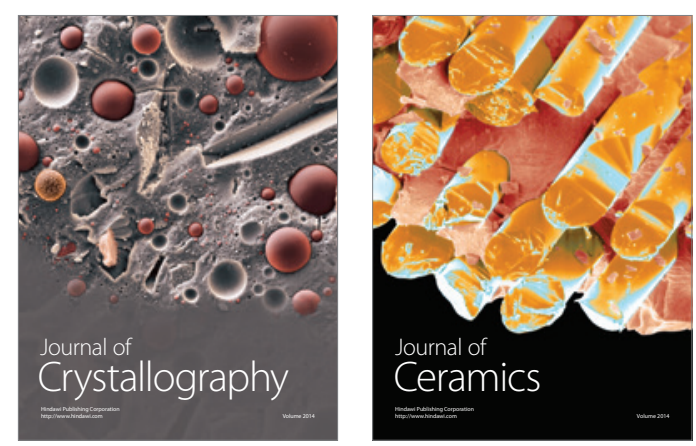
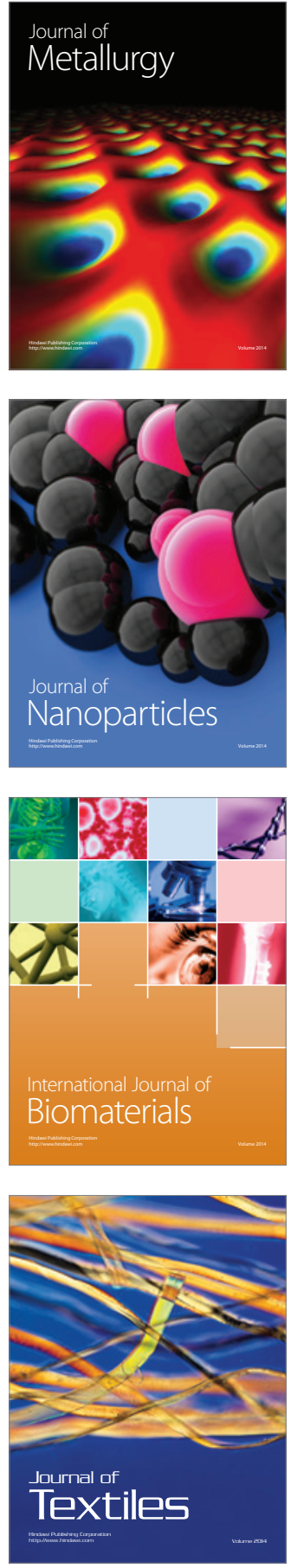